\title{
Diversité et enjeux de conservation des bois sacrés en société Mossi (Burkina Faso) face aux mutations socioculturelles actuelles
}

\author{
Salfo SAVADOGO *, Amadé OUEDRAOGO et Adjima THIOMBIANO \\ Laboratoire de Biologie et Ecologie Végétales, Unité de Formation et de Recherche en Sciences de la Vie et de \\ la Terre (UFR/SVT), Université de Ouagadougou, 09 BP 848 Ouagadougou 09, Burkina Faso. \\ *Auteur correspondant, E-mail : asalfosav@yahoo.fr, Tél: +22678148774 / +22676091008.
}

\section{RESUME}

Au Burkina Faso, la société Mossi a une longue tradition de pratique animiste qui tend à régresser avec la poussée des religions importées comme l'islam et le christianisme. Les vestiges de cette tradition ancestrale que sont les bois sacrés, témoins de l'élément forestier, sont de plus en plus menacés par la forte densité démographique et l'exploitation agropastorale extensive. Afin de montrer les stratégies de gestion et l'impact des facteurs humains et climatiques sur la diversité et la structure de leur peuplement, les bois sacrés ont été étudiés sur la base d'enquête ethnobotanique et de relevés phytosociologiques dans les secteurs sub-sahélien et nord soudanien du Burkina Faso. En société Mossi, les bois sacrés sont caractérisés par la présence en leur sein d'objets traditionnels. Le système de gestion des ressources naturelles repose sur des principes, des règles et des lois coutumières. Cependant, les mutations sociales ont actuellement entraîné l'abrogation ou la détérioration des principes régissant leur protection. Deux cent cinquante (250) espèces appartenant à 130 genres et 70 familles ont été recensées en leur sein. Cette flore est dominée par des phanérophytes $(42,48 \%)$ et des thérophytes $(34,64 \%)$. L'analyse de son spectre phytogéographique montre une dominance des espèces soudaniennes $(28,16 \%)$, des espèces Soudano-zambéziennes $(19,01 \%)$ et des espèces Pantropicales $(18,3 \%)$. Les structures démographiques des peuplements d'arbre dans les bois du secteur nord soudanien montrent des populations stables. Celles dans les bois du secteur sub-sahélien par contre présentent des populations irrégulières.

(C) 2011 International Formulae Group. All rights reserved.

Mots clés: Bois sacrés, enquête ethnobotanique, gestion, diversité, structure, Burkina Faso.

\section{INTRODUCTION}

De nos jours, la gestion des ressources naturelles basée sur les méthodes de conservation modernes a montré ses limites, contrairement aux stratégies traditionnelles de type communautaire basées sur la conservation in situ des aires protégées (Houngnihin, 2005).
L'échec de ces pratiques modernes oblige chaque jour un peu plus à se tourner vers les savoirs locaux (Houngnihin, 2005; Langewiesche, 2006; Deloraine, 2009; Sanou, 2010), vers cette "mémoire millénaire" qu'on a voulu tôt ignorer, voire vilipender, pour y chercher des solutions aux problèmes jugés aujourd'hui inextricables. L'implication de la 
population locale dans la gestion des écosystèmes dévient alors une nécessité impérieuse. Elle est à la base des conventions et des stratégies de conservation de l'environnement. Impliquer et responsabiliser une communauté villageoise riveraine dans l'aménagement et la gestion d'une forêt nécessite que l'on s'inspire de sa tradition qui est un référentiel, un cadre de la pensée et de ses coutumes qui sont une mise en œuvre de la tradition, une capacité de traduire le rêve en réalité. Cette démarche est d'actualité dans un monde en quête de nouveaux repères et où chaque société tente de s'auto représenter dans un souci de donner le meilleur d'ellemême dans la construction d'un monde à visage plus humain et ayant le souci du quotidien des générations à venir (Sanou, 2010).

Les pratiques culturelles dans les aires protégées traditionnelles en Afrique (bois et sites sacrés) sont négligées et souvent rattachées à la superstition par les décideurs politiques. Tous les programmes de gestion durable des politiques environnementales ont échoué du fait de l'évacuation autoritaire des droits coutumiers et de la mise en place de programmes inappropriés dans le contexte Africain (Gadou, 2001; Houngnihin, 2005). Or, les aspects écologiques sont intimement liés aux aspects sociaux et spirituels du système de connaissance (Sow, 2003; Stevensonn, 2005; Langewiesche, 2006; Deloraine, 2009). En effet, Boya (2006) précise ainsi que le savoir écologique traditionnel inclut le contexte socioculturel « invisible » des systèmes de connaissance. Dans le contexte du Burkina Faso où la religion traditionnelle, seule religion gardienne des bois sacrés et pratiquée par environ $40 \%$ de la population (Atlas, 2006), la compréhension des systèmes de gestion des ressources naturelles dans les aires de culte par les scientifiques s'avère nécessaire. Les lieux de culte, en l'occurrence les bois sacrés, sont des réservoirs de diversité biologique et culturelle (Juhé-Beaulaton, 2010), et constituent aussi un facteur de pérennisation de la société rurale (Ballarin, 2006; Cousin et Martineau, 2009; Matthieu, 2010). C'est dans cette optique que la convention sur la biodiversité adoptée au sommet de Rio de Janeiro en 1992, a reconnu la nécessité de protéger les bois sacrés et d'encourager l'utilisation usuelle des ressources biologiques selon les pratiques culturelles et les règles traditionnelles. D'autres rencontres internationales ont été tenues spécifiquement sur les bois sacrés tel le colloque de l'Unesco en 1998 sur le thème «les bois sacrés, la diversité culturelle et la diversité biologique», attestant la prise de conscience de leur importance. Cependant, les connaissances sur les sites sacrés, demeurent relativement sommaires en raison des difficultés liées à leur accès (Bognounou et al., 2001). Les études existantes à ce jour sur les bois sacrés au Burkina Faso (Ouadba, 2003; Traoré, 2007; Savadogo, 2008; Savadogo et al., 2010) se sont moins intéressées aux aspects tels que la diversité des communautés végétales, le mode de gestion des ressources biologiques et les facteurs socioculturels influant leur conservation. La société Mossi, groupe ethnique dominant (sur le plan numérique) du Burkina Faso, a une longue tradition de pratique animiste qui tend à régresser avec la poussée des religions importées comme l'islam et le christianisme. Par ailleurs, les vestiges de cette tradition ancestrale que sont les bois sacrés, témoins de l'élément forestier, sont de plus en plus menacés par la forte densité démographique et l'exploitation agropastorale extensive.

Ce travail s'inscrit dans le cadre de l'évaluation $\mathrm{du}$ potentiel en ressources naturelles et de l'identification des formes d'organisations traditionnelles en matière de conservation des bois sacrés. De façon spécifique, il vise à étudier l'impact du climat sur la diversité biologique et la structure des peuplements, recenser les normes coutumières 
de gestion, répertorier les connaissances et techniques locales de conservation existantes et analyser l'impact des changements socioculturels sur les modes de gestion.

L'étude repose sur l'hypothèse selon laquelle les mutations culturelles constituent une entrave à la conservation de la biodiversité dans les bois sacrés. Le choix de conduire ces travaux en société Mossi se justifie par le fait qu'au Burkina Faso, les Mossi constituent le groupe ethnique majoritaire et ils sont représentés dans toutes les régions du pays avec une forte diversité culturelle. Dans cette société, la population accorde une place très importante aux bois sacrés. Leur étude semble donc intéressante pour mieux analyser et appréhender les rapports entre facteurs sociaux et biodiversité au Burkina Faso.

\section{MATERIEL ET METHODES} Zone d'étude

L'étude a été conduite dans le Plateau Central représenté par les provinces administratives du Sanmatenga, du Namentenga, du Kadiogo, du Bazèga, de l'Oubritenga et du Kourwéogo (Figure 1). Les Mossi qui constituent le principal groupe ethnique de cette partie du pays, représentent $48,6 \%$ de la population totale (Atlas, 2006). Leur religion originelle est l'animisme. Mais de nos jours, on enregistre une forte percée de l'islam, excepté dans la province du Kadiogo où le christianisme domine (Atlas, 2006).

Les provinces les plus septentrionales sont celles du Sanmatenga et du Namentenga $\left(13^{\circ}-14^{\circ} \mathrm{N}\right)$ qui se situent dans le secteur sub-sahélien avec un climat du type sahélien (Guinko, 1984) et une pluviométrie moyenne annuelle comprise entre 600 et $750 \mathrm{~mm}$. La végétation essentiellement dominée par des steppes et des fourrés plus ou moins denses, est constituée d'une flore sahélienne et soudanienne ubiquiste (Thiombiano, 2005). Les quatre autres provinces appartiennent au secteur nord-soudanien $\left(11^{\circ} 30^{\prime} \mathrm{N}-13^{\circ}\right)$, avec des précipitations moyennes annuelles variant entre 750 et $1000 \mathrm{~mm}$ (Thiombiano, 2005). C'est une zone qui abrite différents types de savanes avec une légère dominance des savanes arbustives et arborées (Thiombiano, 2005). Ces deux zones climatiques sont soumises à deux saisons contrastées: une saison des pluies qui se situe entre juin et septembre et une saison sèche allant d'octobre à mai.

\section{Collecte des données}

Les enquêtes sont basées sur des interviews semi-structurées et des causeries informelles conduites avec l'aide d'un guide d'entretien dans différents villages abritant des bois sacrés. En effet, dans tous les villages où nous avons enquêté, l'entretien a été dirigé par un autochtone qui nous montrait les personnes ressources auxquelles nous pouvons nous adresser, après une bonne compréhension des raisons de notre venue et les objectifs de l'étude. Les personnes interrogées sont les autorités coutumières (chefs coutumiers), les gardiens des divinités (chargés de la surveillance des divinités), les notables (conseillers des chefs coutumiers), et d'autres personnes ressources constituées des plus âgées (sans autorité coutumières mais pourvues de connaissances traditionnelles en matière de conservation). $\mathrm{Ce}$ groupe de personnes est supposé mieux indiqué pour fournir des informations pertinentes et fiables sur les statuts sociaux et religieux des bois sacrés ainsi que leur système de gestion. Au total 380 personnes ont été interviewées dans 36 villages. Les enquêtes ont pour objectifs d'étudier les normes coutumières de gestion, répertorier les connaissances et les techniques locales de conservation et à analyser l'impact des changements socioculturels sur les modes de gestion.

L'inventaire floristique de la végétation des bois sacrés a été fait suivant la méthode sigmatiste de Braun-Blanquet, à travers un échantillonnage stratifié pour apprécier la 
diversité floristique des bois sacrés. L'aire minimale a été déterminée dans les deux strates afin de choisir les sites représentatifs et homogènes. C'est la surface minimale dans un tapis végétal pour avoir une image suffisante de ce dernier. Elle permet de tester l'homogénéité de la végétation d'une surface déterminée. Dans cette étude, l'aire minimale varie entre $512 \mathrm{~m}^{2}$ et $1024 \mathrm{~m}^{2}$ d'un bois sacré à un autre pour la strate ligneuse et entre 64 $\mathrm{m}^{2}$ et $128 \mathrm{~m}^{2}$ pour la strate herbacée. La surface d'inventaire dans chaque bois sacré était l'aire minimale correspondante. Dans chaque relevé des ligneux, un relevé des herbacées a été effectué. En cas de forte hétérogénéité, deux ou trois relevés d'herbacées ont été effectués dans la même parcelle. Trois cent cinquante (350) relevés phytosociologiques ont été réalisés dans chaque strate. Les paramètres notés sont les informations générales (le nom de la localité, les coordonnées géographiques, le type de formation, la topographie) et la liste complète des espèces affectées des coefficients d'abondance-dominance selon l'échelle de Braun-Blanquet (1932), modifiée par Wilmanns (1989):

5 : recouvrement supérieur à $75 \%$ abondance quelconque

4 : recouvrement compris entre 75 et $50 \%$ abondance quelconque

3 : recouvrement compris entre 50 et $25 \%$ abondance quelconque

$2 b$ : recouvrement compris entre 25 et $15 \%$

$2 \mathrm{a}$ : recouvrement compris entre 15 et $5 \%$

1 : abondant et recouvrement faible ou peu abondant avec plus grand recouvrement.

+ : simplement présent (recouvrement et abondance très faibles)

L'inventaire dendrométrique s'est fait dans les mêmes parcelles, où les diamètres des ligneux ont été mesurés à hauteur de 1,30 m du sol. Des informations d'ordre général telles la topographie, le type de formation, le type de sol, le nom de la localité et les coordonnées géographiques ont été également notées pour chaque relevé.

\section{Analyse des données}

La répartition centésimale des bois en fonction de leur taille a été réalisée sur Excel 2007. Les avis de la population locale quant à la régression des bois sacrés ont été également analysés à l'aide du même logiciel grâce auquel, les différentes proportions ont été calculées. Les résultats sont présentés sous forme de graphes.

La diversité alpha $(\alpha)$ au sein des bois sacrés a été évaluée par secteur phytogéographique à travers les indices de Shannon-Weiner et l'équitabilité de Piélou afin d'apprécier l'influence du climat sur la diversité biologique. L'indice de diversité (indice alpha) de Shannon- Weiner in Legendre et Legendre (1984) est donnée par la formule:

$H^{\prime}=-\sum_{i=1}^{S} \frac{n i}{N} \log 2 \frac{n i}{N}$

$N i=$ effectif de l'espèce $\mathrm{i} ; N$ effectif total toutes espèces confondues; $S=$ nombre total d'espèces

L'équitabilité de Piélou (1996) est donnée par la formule:

$$
E Q=\frac{H^{\prime}}{\log 2 S}
$$

C'est le rapport de la diversité d'un peuplement ou d'un échantillon à sa diversité maximale. Elle exprime la régularité ou l'équitable répartition des individus au sein des espèces. Elle est comprise entre 0 et 1. Elle tend vers 0 quand la quasi-totalité des effectifs est concentrée sur une espèce, et vers 1 lorsque toutes les espèces ont la même abondance.

Des paramètres dendrométriques (densité et surface terrière) ont été également étudiés pour caractériser les peuplements des bois sacrés par secteur phytogéographique. 
Cela permet d'apprécier l'impact du climat sur la structure des peuplements. La densité a été estimée au nombre d'arbres par hectare. La surface terrière des formations a été calculée selon la formule: $\mathrm{G}=\pi \mathrm{D}^{2} / 4$, exprimée en $\mathrm{m}^{2} /$ ha où $\mathrm{D}=$ diamètre à hauteur de $1,30 \mathrm{~m}$ du sol et $\pi=3,14$. L'analyse des paramètres dendrométriques a été faite sur Excel 2007. Les résultats sont présentés sous forme de tableau (Tableau 2) et de graphe (Figure 9).

Les types biologiques et l'affinité phytogéographique des espèces sont illustrés à l'aide des spectres bruts et pondérés. Les types biologiques désignent l'ensemble des particularités morphologiques qui jouent un rôle dans la résistance aux conditions défavorables, donc dans la localisation des espèces végétales (Guinko, 1984). Les types biologiques (TB) utilisés sont ceux déjà utilisés par divers auteurs pour l'étude des formations végétales tropicales (Adjanohoun, 1964; Sinsin, 1993; Aké Assi, 2002): les thérophytes (Th), les hémicryptophytes (He), les géophytes $(\mathrm{Ge})$, les chaméphytes et les épiphytes (Ep).

Les types phytogéographiques (TP) utilisés sont basés sur les grandes subdivisions chorologiques établies pour l'Afrique (White, 1986) dont les principaux sont: Cosmopolites (Cos), Pantropicales (Pan), Paléotropicales (Pal), Afro-américaines (AA), Soudanozambésiennes (SZ), Afro-tropicales (AT), Afro-malgaches (AM), Plurirégionale Africaine (PA), Guinéo-congolaises (GC), Soudano-guinéennes (SG) et Soudanienne (S). Des spectres pondérés ont été utilisés pour mettre en évidence l'abondance de chaque type biologique ou phytogéographique.

Le spectre pondéré est le rapport en pourcentage entre le recouvrement cumulé des espèces d'un type biologique ou phytogéographique et le recouvrement cumulé des espèces de tous les types biologiques ou phytogéographiques (Sinsin et Oumorou, 2000).
Des analyses de variance (ANOVA) ont été réalisées avec les paramètres de diversité (Richesse spécifique, Indice de Shannon, Equitabilité de Pielou) et dendrométriques (densité et surface terrière). Le secteur phytogéographique et le bois sacré ont été traités comme des facteurs catégoriels fixes afin de rendre compte de la variabilité naturelle dans l'occurrence de l'espèce dans les secteurs phytogéographiques. Toutes les analyses statistiques ont été effectuées dans SAS (SAS Institute Inc., 2008).

\section{RESULTATS}

\section{Caractères généraux}

Les bois sacrés sont en général des formations physionomiquement denses, isolées et très souvent situées à proximité des villages. Dans certaines régions, au nord du Burkina Faso notamment, ils sont identifiables par l'abondance d'espèces remarquables comme Acacia pennata et Combretum micranthum qui forment des fourrés presqu'impénétrables. Ils sont aussi reconnaissables par la présence en leur sein d'anciens objets rituels tels que la poterie, les bagues en fer, les dabas, les pierres taillées, les ruines d'anciennes habitations.

Ils ont une taille moyenne de 1,4 hectare et une minimale de 0,48 hectare. Leur distance moyenne aux agglomérations est de 0,5 kilomètre. Ce sont en générale des fourrés, des savanes arbustives et des savanes arborées. Les fourrés (20\% des bois sacrés) sont des basses formations difficilement penêtrables. Ils sont de trois types: les fourrés monospecifiques à Acacia pennata, les fourrés monospécifiques à Combretum micranthum et les fourrés plurispécifiques constitué essentiellement par ces deux espèces et quelques espèces ibiquistes. Les fourrés sont très fréquents dans le secteur sub-sahélien. Les savanes arbustives sont les plus nombreuses de toute les formations $(50 \%$ des bois sacrés). Elles sont très souvent des peuplements monospécifiques des espèces 
telles Combretum micranthum, Combretum nigricans et Anogeissus leiocarpus. On y trouve également quelques peuplements plurispecifiques. Les savanes arborées sont surtout concentrées dans le secteur nord soudanien et representent $30 \%$ des bois sacrés.

Sur le plan écologique, certains bois sacrés sont associés aux réservoirs, aux mares, aux sources et aux cours d'eau. Nombre d'entre eux sont situés dans des bassins versants près de l'origine d'une source où d'un ruisseau. Ils agissent comme microbassins versants locaux qui permettent de satisfaire les besoins en eau des collectivités locales. D'autres sont représentés par des collines où le sol présente une cuirasse granitique où latéritique.

$\mathrm{Au}$ total 150 bois sacrés ont été inventoriés et se répartissent dans six (06) provinces (Tableau 1). Le plus grand nombre de bois sacrés inventorié se situe dans la province d'Oubritenga en zone nord soudanienne avec une superficie moyenne de 0,53 ha. En effet, la tradition et la culture dans cette province sont toujours de rigueurs et la population accorde beaucoup d'importance aux bois sacrés. La plus forte superficie moyenne a été enregistrée dans la province du Bazèga (3,73 ha). 65,95\% du total de bois inventoriés ont une superficie inférieure ou égale à 1ha tandis que les plus étendus (Superficie $\geq 5$ ha) représentent seulement 2,14\% (Figure 2).

Les Mossi considèrent plusieurs critères pour définir les statuts sociaux des bois sacrés:

- suivant le propriétaire, on distingue les bois sacrés personnels, les bois sacrés familiaux, les bois sacrés claniques et les bois sacrés communautaires;

- selon les mesures conservatoires il existe les bois sacrés intégralement protégés (prohibition de toute activité en leur sein) et les bois sacrés à protection non intégrale (autorisation de prélèvement à des fins précis en cas d'absence d'une espèce dans les terroirs);

- selon ce qu'ils abritent, on distingue les bois de divinités (abritant des divinités), les bois cimetières (abritant des tombeaux physiques et mystiques), les bois divinitécimetières (abritant des divinités et des cimetières).

\section{Approche locale de la gestion des ressources naturelles}

Le système de gestion des ressources naturelles des bois sacrés chez les Mossi repose sur des principes, des règles et des lois coutumières (pas de coupe de bois, pas de feu, pas de chasse, pas de fréquentation en dehors des jours de culte). Dans la tradition animiste, chaque quartier dispose au moins d'un bois sacré. Les personnes responsables en charge de la gestion sont principalement les autorités coutumières, les gardiens des divinités, les notables et d'autres personnes ressources constituées des plus âgées. Les agents des eaux et forêts s'impliquent très rarement dans la gestion des bois sacrés car leur intervention est souvent perçue par les habitants comme une immixtion dans leurs affaires intimes. Ils interviennent plutôt dans la gestion des forêts communautaires villageoises qui n'ont aucun caractère sacré. La gestion des bois sacrés qui est directement assurée par la population locale répond au souci de préserver les coutumes, les traditions et les «secrets» du village.

Des sanctions sont prises par les autorités coutumières, à l'encontre des contrevenants (amende de cola, de dolo, de mouton, de chèvre, de bœuf). Ces amendes permettent aux contrevenants de présenter leur excuse aux êtres surnaturels offensés dans les bois. S'en suivra la mort du contrevenant si celui-ci refuse de payer cette amende. Des malédictions sont également formulées contre tous transgresseurs aux lois et aux principes coutumiers. Ces malédictions se font dans certains bois sacrés réputés hantés, à travers 
des sacrifices et des incantations. Nous citons en exemple le lieu de culte dénommé Bangtodo à Yimdi dans la province du Kadiogo. Les malédictions sont réparables jusqu'à des dates préalablement fixées par les responsables coutumiers. Si le contrevenant reconnaît ses faits et vient demander des excuses auprès des féticheurs, ils l'amendent et font des sacrifices de désenvoûtement. Cependant, les malédictions deviennent irréparables lorsque le condamné fait fit des malédictions et les laisse traîner au delà des délais préétablis.

Des sacrifices rituels liés à la vie du village sont faits à des moments précis de l'année, dans les lieux de cultes à des fins diverses (pluies, bonnes saison, santé de la population...). En début de saison de pluie par exemple, chaque chef de terre fait des sacrifices pour que les ancêtres octroient aux habitants de très bonnes récoltes et, en fin de saison, la population se doit de remercier à travers d'autres sacrifices. En cas de sécheresse, des sacrifices sont également faits dans certains bois sacrés pour demander de la pluie aux ancêtres.

Dans certains villages, toute activité est interdite dans les bois par la loi coutumière même le ramassage de bois mort. Dans d'autres villages les prélèvements à des fins médicinales, la cueillette des fruits, la fauche d'herbe et le ramassage de bois mort sont autorisés dans les bois sacrés par le responsable des sacrifices. Mais celui-ci ne donne son accord que si les plantes recherchées n'existent plus dans les brousses environnantes. Le ramassage de bois mort lorsqu'il est autorisé, ne concerne que les personnes très âgées incapables de se rendre en brousse. Des initiatives de gestion moderne sont prises par la population locale au nombre desquelles:

- la délimitation des bois sacrés par des clôtures en grillage, des haies vives, des diguettes et des piquets en béton ;
- l'enrichissement artificiel des bois à base d'espèces locales et exotiques;

- l'usage de panneaux de signalisation indiquant la dénomination du bois, les activités interdites, la mise en défens;

- Le désherbage des contours des bois sacrés pour les préserver du feu.

\section{La gestion des bois sacrés dans le contexte socioculturel actuel}

$\mathrm{Au}$ Burkina Faso en général et en société Mossi en particulier, l'animisme était la seule religion jadis pratiquée. La tradition et la coutume étaient de rigueur dans beaucoup de tribus Mossi et les dignitaires animistes veillaient rigoureusement sur la préservation des bois sacrés qui abritent souvent les divinités adorées. Mais de nos jours, l'entrée du christianisme et de l'islam a entraîné des mutations sociales qui ont affecté beaucoup de cultures, notamment celle des Mossi. Ces mutations se sont traduites par des conversions massives en ces religions monothéistes et par conséquent l'abandon progressif des pratiques et des rites animistes. S'en est suivi le non respect et le rejet de nombreuses lois coutumières avec les changements des manières de penser et de comportements. Les cultes ne sont désormais connus et pratiqués que par de rares personnes animistes d'un âge avancé.

Dans les localités fortement islamisées, il y a très peu ou pas de bois sacrés. Les seules formations végétales présentant toujours un caractère sacré sont les lieux hantés, c'est-àdire les bois supposés abriter des esprits maléfiques. De l'avis des populations locales, les facteurs qui sont à l'origine de la régression des formations sacrées sont principalement l'implantation des religions étrangères, l'occupation anarchique des terres à des fins agricoles, la demande croissante en ressources naturelles (bois d'énergie, de construction, plantes médicinales...) entraînant souvent des prélèvements 
anarchiques, le pâturage, la modernisation et l'urbanisation qui entraînent le déguerpissement de certaines populations qui abandonnent ainsi de nombreux lieux de culte (Figure 3).

\section{Etat de conservation des bois sacrés Diversité floristique}

Deux cent cinquante (250) espèces au total ont été recensées. Ces espèces sont réparties en 130 genres et 70 familles dont les plus représentées dans la flore ligneuse sont les Combretaceae (17\%), les Mimosaceae (15\%), et les Ceasalpiniaceae (12\%) (Figure 4). Chez les herbacées, les familles les plus représentées sont les Poaceae (30\%), les Fabaceae (18\%) et les Caesalpiniaceae $(7 \%)$ (Figure 5). Les richesses spécifiques moyennes des bois varient significativement d'un secteur phytogéographique à l'autre ( $\mathrm{p}=$ $0,03)$. Les indices de Shannon $(p=0,065)$ et l'équitabilité de Piélou ( $\mathrm{p}=0,068)$ ne varient pas significativement d'un secteur à l'autre.

Les types biologiques prédominants sont les phanérophytes et les thérophytes, avec une légère abondance-dominance des premières sur les secondes (Figure 6). Les géophytes $(7,18 \%$ de spectre brute), les hémicryptophytes $(6,53 \%$ de spectre brute), les chaméphytes $(5,8 \%$ de spectre brute), les épiphytes $(1,3 \%$ de spectre brute) sont très peu nombreuses. Les spectres pondérés suivent en général la même tendance. Une analyse du spectre des sous-types de phanérophytes montre une prédominance des microphanérophytes $(60 \%$ de spectre brute et $75 \%$ de spectre pondéré) (Figure 7).

Le spectre phytogéographique global (Figure 8) présente une forte proportion de l'élément-base Soudanien $(28,16 \%$ de spectre brut et $35,4 \%$ de spectre pondéré), suivi des espèces Soudano-zambéziennes $(19,01 \%$ de spectre brute et $15,79 \%$ de spectre pondéré), des espèces Pantropicales (18,3\% de spectre brute et $15,28 \%$ de spectre pondéré) et les espèces Paléotropicales $(11,26 \%$ de spectre brute et $15,6 \%$ de spectre pondéré). Globalement, il y a une forte proportion des espèces à distribution continentale.

\section{Structure démographique des peuplements}

Dans le secteur sub-sahélien, on enregistre des structures de population instables, caractérisées par une faible proportion d'individu dans la classe [15-20 $\mathrm{cm}$ [ et une absence totale d'individu dans la classe [10-15 cm[ (Figure 9). Cela peut être dû à leur écologie car certains bois sont représentés par des collines dont les conditions édaphiques (cuirasse granitique ou latéritique) ne favorisent pas le développement des espèces. Dans ce secteur, les espèces sont également soumises à une pression humaine (coupes sélectives et clandestines de bois) en dépit des interdits traditionnels, ce qui peut expliquer les faibles proportions d'individus dans certaines classes.

La distribution par classe de diamètre des peuplements d'arbre dans le secteur nord soudanien est illustrée par l'histogramme en forme de «L» (Figure 9). Cette distribution montre des structures de populations stables, caractérisées par la présence des individus dans presque toutes les classes de diamètre. En plus, un grand nombre de sujet se trouve dans la classe [5-10[. Ce secteur étant plus arrosé que le sub-sahélien, les espèces bénéficient de très bonnes conditions d'humidité. En plus, les pressions anthropiques sont faibles dans ce secteur.

La densité moyenne des bois sacrés varie significativement d'un secteur à l'autre $(\mathrm{p}=0,0001)$. Les plus grandes densités $(8850$ $\pm 63,79)$ et surfaces terrières $(32,9 \pm 0,66)$ sont rencontrées dans les bois du secteur nord soudanien (Tableau 2). La surface terrière moyenne varie aussi significativement d'un secteur à l'autre (p=0,0001). 


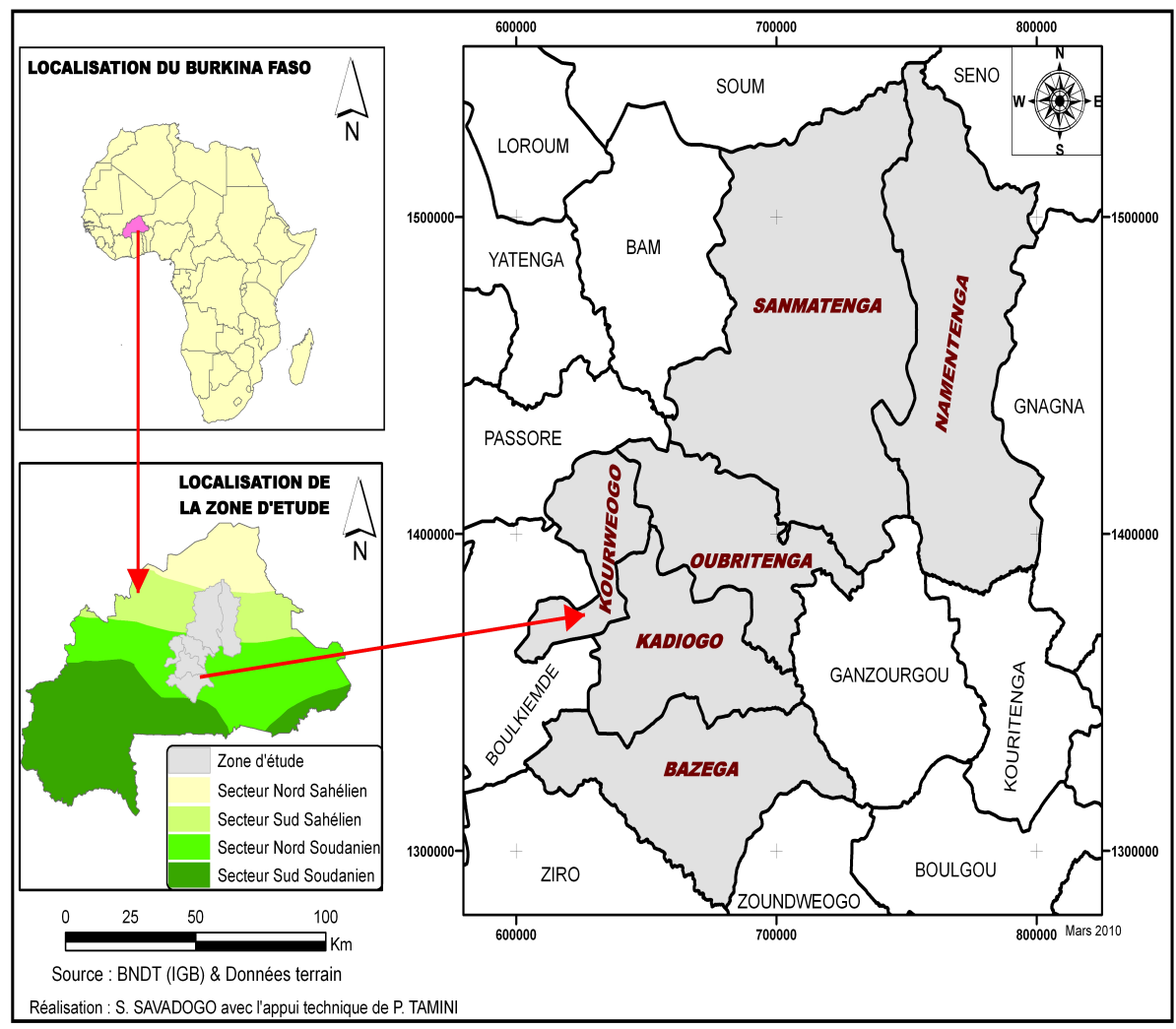

Figure 1: Localisation de la zone d'étude.

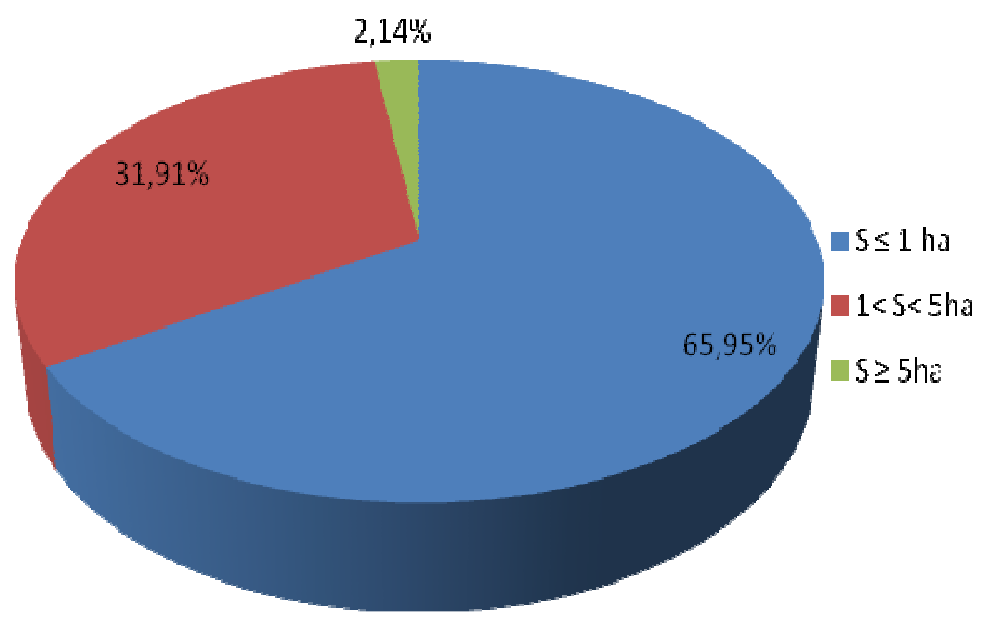

Figure 2: Répartition centésimale des bois sacrés en fonction de leur taille ( $\mathrm{s}=$ superficie, ha= hectare). 


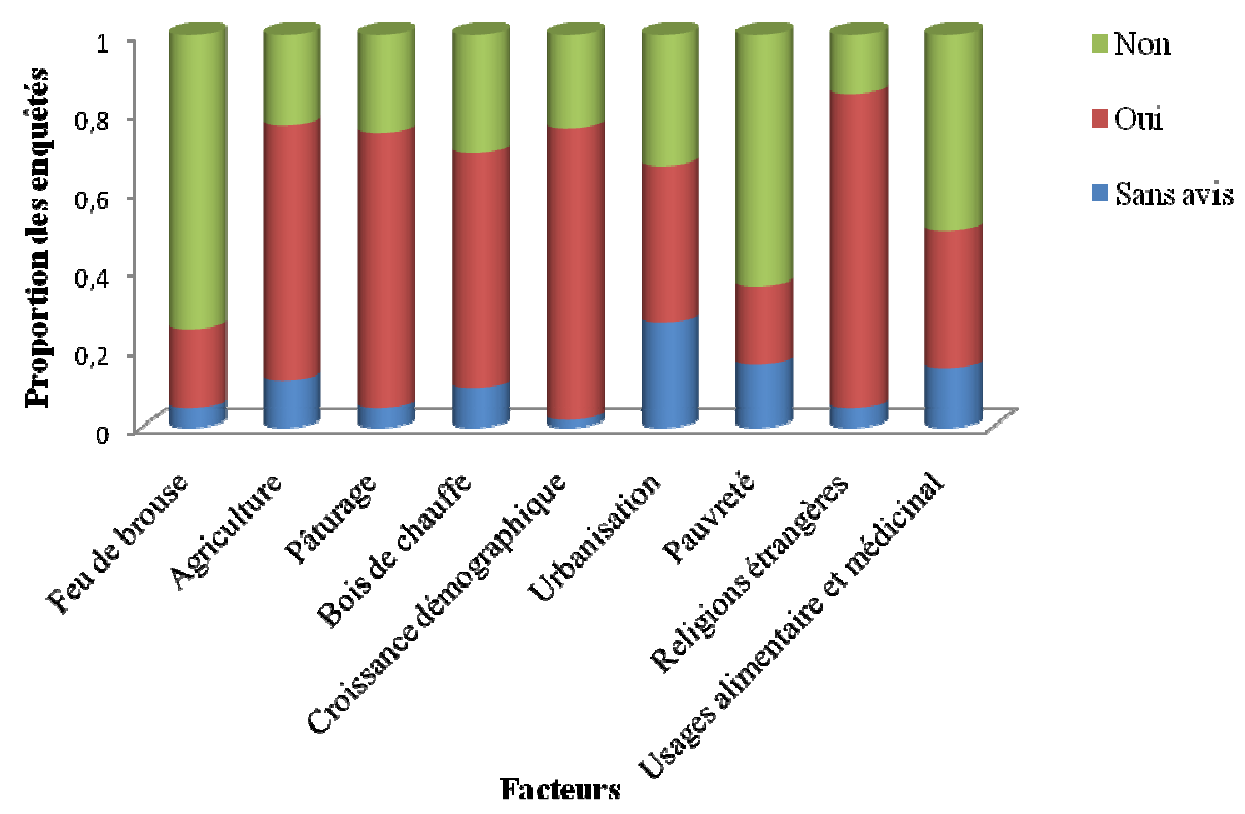

Figure 3: Facteurs de dégradation des bois sacrés selon les personnes enquêtées.

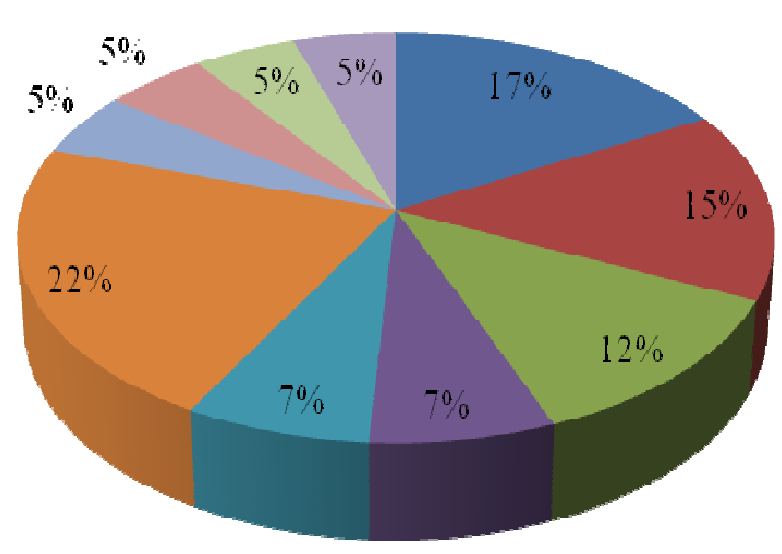

- Combretaceae

- Minnosacea

- Cassalpiniaceae

- Capparidaceae

- Rubiaceae

- Autres familles

Tiliaceae

- Aldaradiateare

- Moraceae

Fabaceae

Figure 4: Spectre des principales familles de la flore ligneuse des bois sacrés. 


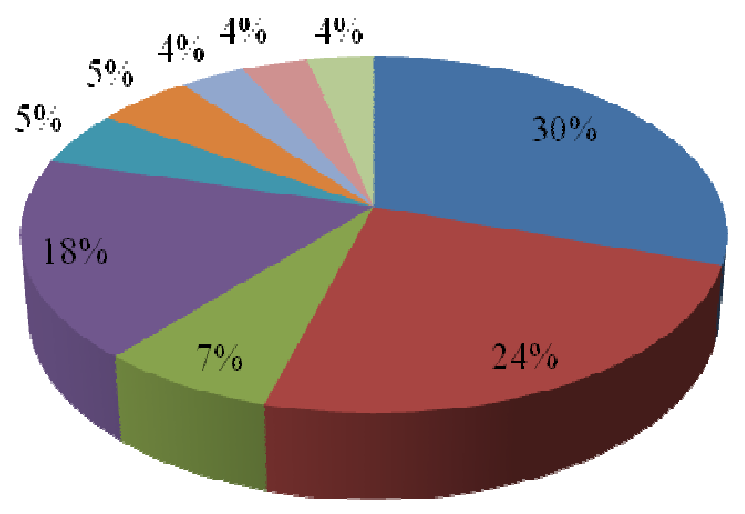
- Poaceae
- Autros famillos
- Coesalpiniacene
- Habaceae
-Convolvulaceae
-Tiliaceae
Euphorbiaccac
- Allamililiacente
Nalvaceae

Figure 5: Spectre des principales familles de la flore herbacée des bois sacrés.

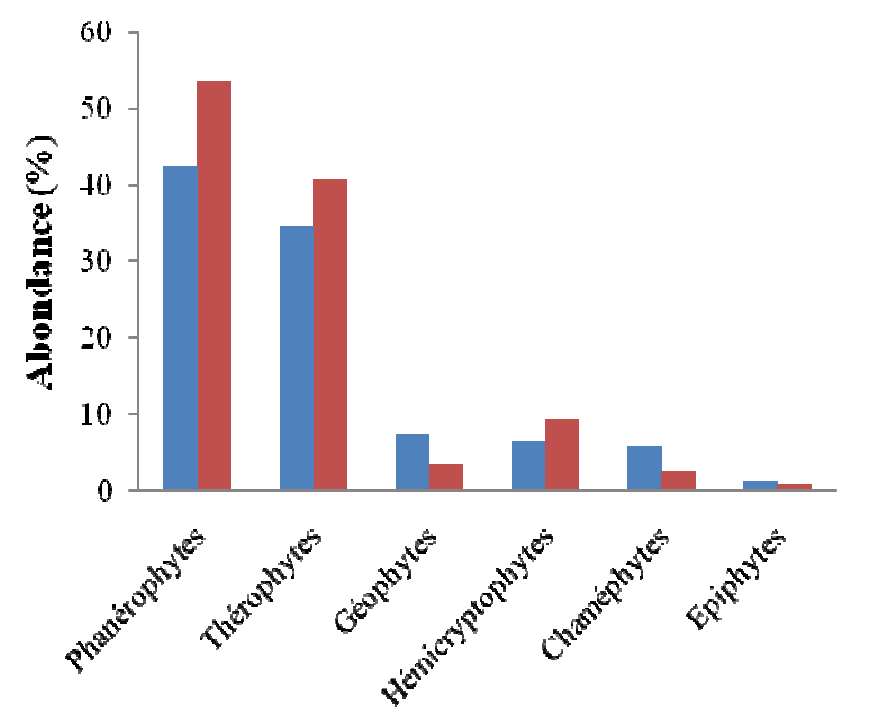

- Spectre 10inte

- Spectie pondere

\section{Typesbiologiques}

Figure 6: Spectre des types biologiques des bois sacrés. 


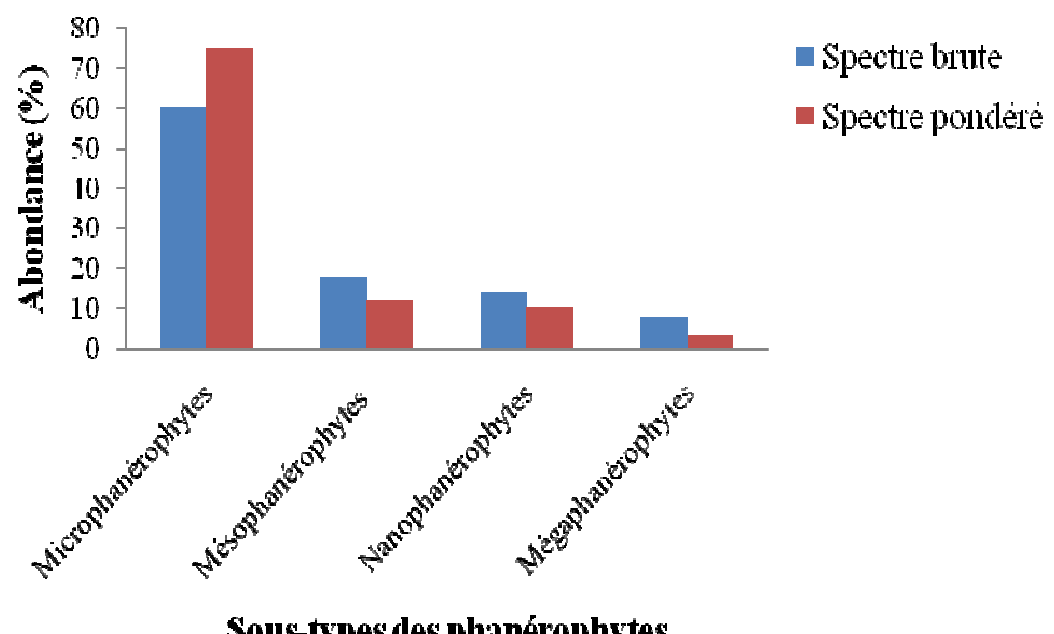

Figure 7: Spectre des sous-types de phanérophytes.

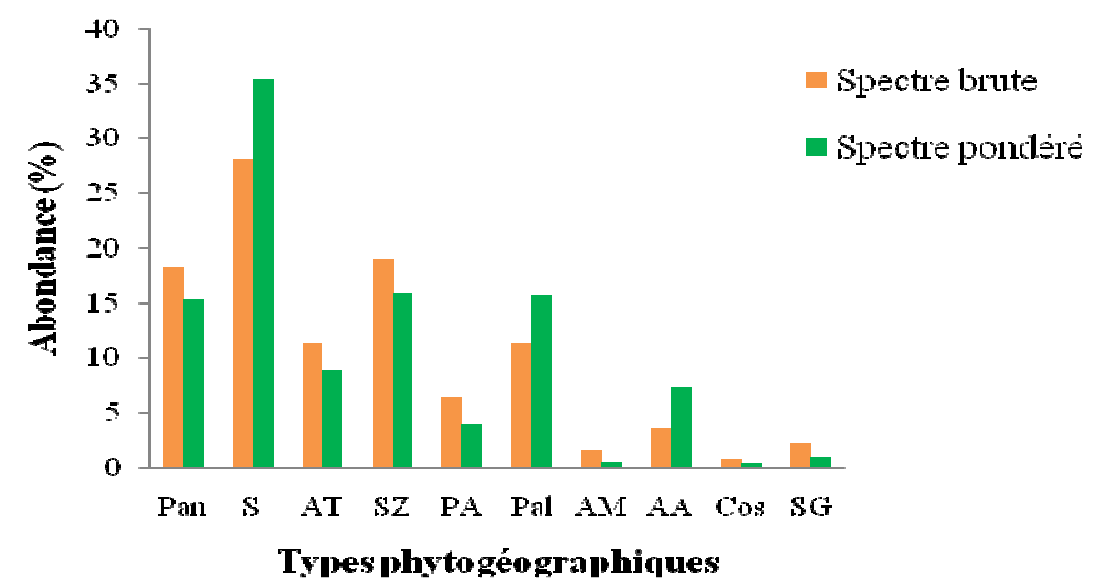

Figure 8: Spectre des types phytogéographiques des bois sacrés.

Pan: Pantropicale; S: Soudanienne; AT: Afro-tropicale; SZ: Soudano-Zambézienne ; PA: Plurirégionale Africaine; Pal: Paléotropicale, AM: Afro-malgache; AA: Afro-américaine; Cos: Cosmopolite; SG: Soudano-guinéenne. 


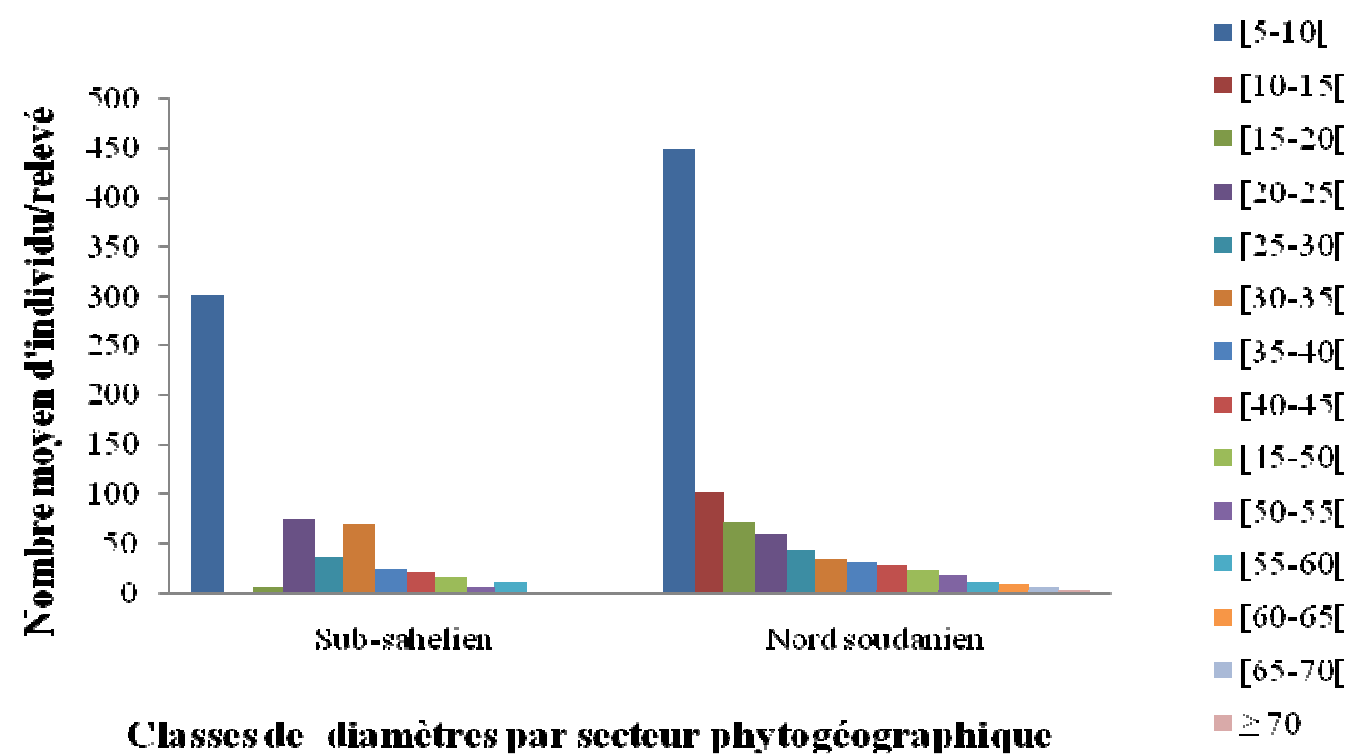

Figure 9: Structure globale par classe de diamètre des peuplements d'arbres par secteur phytogéographique. 
S. SAVADOGO et al. / Int. J. Biol. Chem. Sci. 5(4): 1639-1658, 2011

Tableau 1: Taille et répartition des bois sacrés par province et par zone phytogéographique.

\begin{tabular}{lllllll}
\hline Secteur phytogéographique & \multicolumn{2}{l}{ Nord soudanien } & \multicolumn{3}{l}{ Sub-sahélien } \\
\hline Provinces & Kadiogo & Oubritenga & Bazèga & Kourwéogo & Sanmatenga & Namentenga \\
Nombre de bois sacrés & 30 & 35 & 20 & 21 & 24 & 20 \\
Superficie moyenne des bois (ha) & 1,55 & 0,53 & 3,78 & 0,48 & 1,35 & 0,73 \\
\hline
\end{tabular}

Tableau 2: Densité et diversité floristique des bois sacrés par secteur phytogéographique.

\begin{tabular}{lcc}
\hline & Nord soudanien & Sub-sahélien \\
\hline Nombre de relevés & 175 & 175 \\
Densité moyenne (tiges/ha) & 8850 & 6500 \\
Surface terrière moyenne $\left(\mathrm{m}^{2} / \mathrm{ha}\right)$ & 32,9 & 25,32 \\
$\begin{array}{l}\text { Richesse spécifique moyenne } \\
\text { (nombre d'espèce/relevé) }\end{array}$ & 35 & 25 \\
Indice de Shannon & 3,18 & 2,78 \\
Equitabilité de Piélou & 0,93 & 0,92 \\
\hline
\end{tabular}




\section{DISCUSSION}

Importance socio-culturelles des bois sacrés

Comme l'ont souligné Juhé-Beaulaton et al. (2005), Dhaila et Bhupendra (2007), Kokou et Kokutse (2007), Sukumara et Jeeva (2008), Ceperley et al. (2010), les bois sacrés ont une grande importance socio-culturelle. En effet, ils abritent des divinités, des cimetières et sont aussi des lieux d'initiation et de célébration de cérémonies rituelles. Selon Kokou et Kokutse (2007), les Ewé, peuple originaire de Tado au Togo, pratiquent le culte Vaudou dans lequel la forêt et les espèces végétales tiennent une place importante, comme abris à de nombreuses divinité ou comme leurs symboles. Nos résultats sont également consolidés par ceux de Garcia et al. (2006) qui en Inde, décrivent les forêts sacrées comme des fragments forestiers associés à un esprit, une divinité ou un temple et comme des écosystèmes préservés en raison des croyances et pratiques qui y sont associées. En outre, Crews (2003) soutient les propos de ces auteurs par ces mots: « depuis la nuit des temps, la nature et les arbres en particulier ont été pour les communautés prémonothéistes, source d'inspiration fondée sur la perception de l'arbre comme symbole de la vie donnée par les divinités». Les bois sacrés représentent des symboles identitaires des différentes sociétés (Cousin et Martineau, 2009). La catégorisation des bois sacrés selon ce qu'ils abritent (bois cimetières, bois de divinités) et suivant leur propriétaire (bois familiaux, bois de quartier et bois communautaires) en pays Mossi est comparable à celle faite par Kokou et Sokpon (2006) dans leur étude sur les forêts sacrées du couloir de Dahomey au Bénin. Les sites sacrés sont des conservateurs des pratiques traditionnelles et offrent à la population des avantages multiples. La tradition confère à la communauté villageoise son identité et sa personnalité et lui donne la capacité de relever les grands défis qui se présentent à chaque époque (Sanou, 2010).
C'est au nom de ces pratiques que périodiquement les habitants se rendent sur les autels communautaires dans les forêts pour solliciter aux esprits de la brousse et à ceux des ancêtres des bénédictions en début de saison de pluies ou à la fin des récoltes pour les remercier.

\section{Approche locale de gestion des bois sacrés}

La gestion traditionnelle des ressources naturelles dans les bois sacrés, basée sur les interdits coutumiers, les principes et les totems met beaucoup d'espèces animales et végétales à l'abri d'exploitation. De ce faite, ces formations deviennent des lieux de refuges pour la diversité biologique. En effet, nul n'a le droit d'y entretenir un champ, d'y chasser, encore moins d'aller y couper ou ramasser du bois de chauffe (Juhé-Beaulaton, 2010). Celui qui, sans autorisation du chef de village (première autorité coutumière $\mathrm{du}$ village), va prélever dans une forêt sacrée quelque menu produit pour ses besoins quotidiens, exposerait tous les habitants à des maux supposés générés par le courroux des esprits des ancêtres fondateurs du village, en l'occurrence la prolifération des moustiques, la sécheresse, les maladies incurables... L'exemple du bois sacré de Goupana dans la province du Kadiogo est assez révélateur.

L'importance des interdits et des règles coutumières dans la conservation de la biodiversité a été mis en évidence au Burkina Faso (Bognounou et al., 2001), en Côte d'Ivoire (Gadou, 2001; Ibo, 2005) et en Inde (Swamy et al., 2003; Ashalata et al., 2005). De même, Madeweya et al. (2009) ont montré que les espèces du genre Ficus sont réputées abriter des esprits et sont pour cela épargnées de la coupe.

\section{Facteurs de dégradation des bois sacrés} Malgré les systèmes d'interdits, de nombreux problèmes apparaissent autour des bois sacrés. Selon Caillault et al. (2010) et Matthieu (2010), ces problèmes concernent 
essentiellement deux points: la diminution de la superficie de l'espace boisé, provoquée par la forte pression sur les terres, et la surexploitation des ressources de la forêt. La coupe clandestine de bois dévient de plus en plus grandissante malgré les interdits traditionnelles, et cela par la forte demande de bois de chauffe, surtout en milieu urbain. Les énergies traditionnelles (bois et charbon de bois) représentent $89 \%$ de la consommation des ménages ruraux et urbains (Yelkouni, 2005).

L'introduction de nouvelles confessions religieuses (Islamique et Chrétienne) dont certaines idéologies s'opposent fortement aux pratiques des rites traditionnels jugés sataniques et démoniaques, a aussi ébranlé la cohésion sociale et contribué à la fragilisation des forêts sacrées, menaçant ainsi leurs existences. Cela tient du fait que l'établissement et la protection des forêts sacrées étaient principalement basés sur des croyances culturelles et religieuses locales. Juhé-Beaulaton (2005), Kokou et al. (2005) ont également mis en évidence plusieurs facteurs qui contribueraient à la dégradation de la diversité biologique dans les bois sacrés. Il s'agit de la croissance démographique, de l'urbanisation, de l'introduction des religions monothéistes. Un autre facteur de menace pour les bois sacrés est le faible droit de propriété (Ishani et William, 2009).

La régression des pratiques ancestrales liées aux bois sacrés peut cependant, avoir d'énormes conséquences pour la société, notamment rurale. En effet, une étude très récente faite par Traoré et al. (2011), montre que de l'avis de certains Senoufo du Burkina Faso, l'abandon des traditions ancestrales est l'une des causes de la baisse de la pluviométrie.

\section{Diversité floristique des bois sacrés}

L'inventaire floristique a permis de recenser 250 espèces dans les 150 bois sacrés. Les bois sacrés du secteur nord soudanien sont les plus riches floristiquement. Ce qui se traduit par un indice de diversité de Shannon (indice alpha) relativement élevé $(3,18)$. Cela s'explique d'une part par l'effort de conservation des populations de la zone et l'influence climatique d'autre part. Selon Thiombiano (2005) les précipitations moyennes annuelles de ce secteur varient entre 750 et $1000 \mathrm{~mm}$. Ce qui peut considérablement influencer la composition botanique des bois sacrés. Le spectre biologique montre une prédominance des thérophytes et des phanérophytes. Ces résultats sont similaires à ceux d'autres auteurs qui ont travaillé dans des aires protégées en zone nord soudanienne du Burkina Faso (Gnoumou, 2007; Mbayngone, 2008; Mbayngone et al., 2008). La dominance des thérophytes montre en partie la prédominance des herbacées dans les bois sacrés. Cela s'explique par leur grand pouvoir de dissémination. Les microphanérophytes sont le sous type dominant au sein des phanérophytes. Des études récentes effectuées par Sambaré et al. (2010) dans des formations ripicoles du Burkina Faso ont montré des résultats similaires. La végétation des bois sacrés en société Mossi est dominée par des combretaceae. Sa flore est majoritairement constituée d'espèces Soudaniennes, suivi des espèces Soudano-zambéziennes, des espèces Pantropicales et les espèces Paléotropicales. Ce qui explique l'affinité de ces espèces au secteur sub-sahélien et nord soudanien. La prédominance de l'élément-Base Soudanien et des Soudano-zambéziennes sur les autres types phytogéographiques est une caractéristique des savanes soudaniennes (Guinko, 1984).

\section{Structure des peuplements}

La distribution des individus par classe de diamètre présente des courbes d'allure en $\mathrm{L}$ dans les bois sacrés du secteur nord soudanien. Selon Ouédraogo (2006), une telle distribution caractérisée par un grand nombre 
de bois de petit diamètre et un nombre très restreint de bois de gros diamètre, est typique des populations stables, susceptibles de se renouveler par la régénération naturelle. Ces distributions sont semblables à celles obtenues par Fongnzossie et al. (2008) dans les peuplements d'arbre du sanctuaire à gorille au sud Cameroun. Selon les auteurs de cette étude, tout peuplement forestier non perturbé montrerait une distribution de ce type. En effet, les cas de coupes et de mutilations n'ont pas été constatés sur le terrain. Ce qui pourrait justifier la bonne structure et la forte densité des peuplements (8850 pieds/ha). Cette bonne structure des peuplements pourrait également s'expliquer par la pluviométrie annuelle de la zone relativement forte ( 750 à $1000 \mathrm{~mm} / \mathrm{an})$.

Dans les peuplements des bois du secteur sub-sahélien par contre, les structures de population irrégulières ou vieillissantes montrent une instabilité de cette population et les difficultés pour un renouvellement éventuel. Cette mauvaise structure des peuplements serait due aux difficiles conditions édaphiques et climatiques d'une part et à la pression anthropique sur certains bois sacrés d'autre part. Des études ont montré que les effets des sécheresses répétées dans les années 1970 et 1980, notamment en Afrique, ont directement augmenté la mortalité des populations ligneuses des écosystèmes sensibles (Adjonou et al., 2009). Un grand nombre de bois en zone subsahélienne est en outre représenté par des collines dont les conditions édaphiques souvent difficiles ne permettent pas le développement des espèces. Ce qui explique le faible nombre d'individu dans certaines classes de diamètre et l'absence totale d'individus dans la classe [10-15 cm[. C'est dans ce sens que Awokou et al. (2009) précisent que le sol et la topographie jouent un rôle capital dans le déterminisme des diverses phytocenoses. L'absence d'individu dans la classe [10-15 cm[ est également due à une forte mortalité des individus suite à des pathologies et à des dégénérescences naturelles. En effet, les mortalités des arbres liées aux événements tels que la dégénérescence naturelle ou les infections pathologiques ont été décelées sur le terrain.

\section{Conclusion}

Les bois sacrés constituent, sans conteste, des lieux de refuge pour de nombreuses espèces. En effet, les interdits traditionnels et la perception favorable qu'ont les habitants quant au caractère mystique de certaines espèces sont des atouts pour une bonne protection des bois sacrés et une conservation des espèces, voire des ressources naturelles locales en général. Mais avec la pression foncière actuelle et les mutations socioculturelles induites par la forte implantation des religions monothéistes (Islam et christianisme), beaucoup d'interdits coutumiers, principes et totems impliquant la préservation des espèces et des habitats sont en forte régression. Ce qui expose les ressources naturelles à une exploitation anarchique. Il est alors nécessaire d'entreprendre des actions suivantes:

- intégrer les acquis de la législation coutumière dans la législation moderne de conservation de l'environnement;

- légiférer et promulguer des lois en faveur des sites sacrés;

- préserver et mettre l'accent sur les traditions.

\section{REMERCIEMENTS}

Nous remercions vivement le Projet BIOTA (Biodiversity Monitoring Transect Analysis) pour avoir entièrement financé nos travaux et les autorités coutumières pour leur confiance.

\section{REFERENCES}

Adjanohoun E. 1964. Végétation des Savanes et des Rochers Découverts en Côte d'Ivoire. Centre ORSTOM: Paris ; 180.

Adjonou K, Bellefontaine R, Kokou K. 2009. Les forêts claires du Parc national OtiKéran au Nord-Togo: structure, 
dynamique et impacts des modifications climatiques récentes. Sécheresse, 20(1): 110.

Aké Assi L. 2002. Flore de la côte d'Ivoire: catalogue systématique, biogéographie et écologie. Boissiera, 58: 401.

Ashalata DK, Khan ML, Tripathi RS. 2005. Sacred grove of Manipur, northeast India: Biodiversity value, status and strategies for their conservation. Biodiversity and Conservation, 14: 1541-1582.

Atlas. 2006. Atlas du Burkina. P. 54.

Awokou KS, Ganglo CJ, Azontondé HA, Adjakidjè V, Foucault DB. 2009. Caractéristiques structurales et écologiques des phytocénoses forestières de la forêt classée d'Itchèdè (Département du Plateau, Sud-est Bénin). Sciences \& Nature, 6(2): 125 - 138.

Ballarin MP. 2006. Culte des ancêtres royaux et légitimation du pouvoir dans la région de Majunga (nord-ouest de Madagascar). Journal des Anthropologues, 104: 191217.

Bognounou O, Belem OM, Lamien N. 2001. Connaissances et pratiques traditionnelles pour une conservation de la biodiversité au Burkina Faso. In Pratique Culturelles, la Sauvegarde et la Conservation de la Biodiversité en Afrique de l'Ouest et du Centre. Collection de l'atelier: Ougadougou ; 105-121.

Boya BM. 2006. Des stratégies intégrées durables: Savoir écologique traditionnel et gestion adaptatives des espaces et des ressources. Vertigo, 7(2): 1-10.

Braun-Blanquet J. 1932. Plant Sociology. Macgran-Hill: New York \& London; p. 330.

Caillault S, Delahaye D, Ballouche A. 2010. Des cultures temporaires face à la forêt classée. Exemples des paysages à l'ouest du Burkina Faso, projet de paysage, p. 13.

Ceperley N, Montagnini F, Natta A. 2010. Importance des sites sacrés pour la conservation des forêts-galeries au centre-
Benin. Bois et Forêts des Tropiques, 303(1): 5-23.

Cousin S, Martineau JL. 2009. Le festival, le bois sacré et l'UNESCO. Logiques politiques du tourisme culturel à Osogbo (Nigeria). Cahiers d'Etudes Africaines, 1(193): 337-364.

Crews J. 2003. Forest and tree symbolism in folrklore. Unasylva, 54(213): 1-7.

Deloraine L. 2009. Place de l'arbre dans la mythologie Germano-scandinave. Tela Botanica Association.

Dhaila-Adhikari S, Bhupendra SA. 2007. Veneration of a deity by restoration of sacred grove in a village Minar, Kumaun region of Uttarakhand: A case study. Journal of American Science, 3(2): 1-5.

Fongnzossie FE, Tsabang N, Nkongmeneck BA, Nguenang GM, Auzel P, Christiana E, Kamou E, Balouma JM, Apalo P, Mathieu H, Valbuena M, Valère M. 2008. Les peuplements d'arbres du Sanctuaire à gorilles de Mengamé au sud Cameroun. Tropical Conservation Science, 1(3): 204221.

Gadou DM. 2001. Préservation des religions africaines: les réponses des religions africaines. In Pratique Culturelles, la Sauvegarde et la Conservation de la Biodiversité en Afrique de l'Ouest et du Centre. Collection de l'atelier: Ouagadougou ; 49-67.

Garcia C, Pascal JP, Kushalappa CG. 2006. Les forêts sacrées de Kodagu en Inde: écologie et religion. Bois et Forêts des Tropiques, 2(288): 1-9.

Gnoumou A. 2007. Etude de la flore et de la végétation du parc urbain Bangr-weoogo. Mémoire de DEA, Université de Ouagadougou, p. 71.

Guinko S. 1984. Végétation de la Haute Volta. Thèse de Doctorat ès Sciences Naturelles, Univ. Bordeaux III, p. 394.

Hounghihin AR. 2005. Les Mécanismes Endogènes dans la Problématique de l'Environnement à Covè (Benin). 
CODESRIA : Maputo (Mozambique) ; 118.

Ibo J. 2005. Contribution des organisations non gouvernementales écologistes à l'amelioration des forêts sacrées en Côte d'Ivoire: l'expérience de la croix verte. Vertigo, 6(1): 1-13.

Ishani P, William BJR. 2009. A socioecological study of sacred grooves and Memorials Park: Case from USA and India.

Juhé-Beaulaton D. 2005. Enjeu économique et sociaux autour des bois sacrés et «la conservation de la biodiversité» Benin, Burkina Faso et Togo. Actes de l'atelier Paris.

Juhé-Beaulaton D, Boutais J, Roussel B. 2005. Les sites religieux vodun: Des patrimoines en permanente évolution. MC. Cornier-Salem.

Juhé-Beaulaton D. 2010. Forêts sacrées et sanctuaires boisés. Des créations culturelles et biologiques (Burkina Faso, Togo, Bénin), Paris, Karthala.

Kokou K, Adjossou K, Hamberger K. 2005. Les forêts sacrées de l'aire Quatchi au sud-est du Togo et les contraintes actuelles des modes de gestion locales des ressources forestières. Vertigo, 6(3): 1-10.

Kokou K, Kokutse AD. 2007. Conservation de la biodiversité dans les forêts sacrées littorales du Togo. Bois et Forêts des Tropiques, 292: 59-72.

Kokou K, Sokpon N. 2006. Les forêts sacrées du couloir de Dahomey. Bois et Forêts des Tropiques, 2(288): 15-23.

Langewiesche K. 2006. La forêt, les ancêtres et le marché. Perceptions locales de la forêt et de ses changements au NordBénin. Afrika Spectrum, 2(41): 221-248.

Legendre L, Legendre P. 1984. Ecologie Numérique. Le Traitement Multiple des Données Ecologiques (Tome 1). Masson: Paris.

Madeweya KH, Oka H, Matsumoto M. 2009. Sustainable management of sacred forest and their potential for eco-tourism in Zanzibar. Bulletin of FFPRI, 3(390): 3348.

Matthieu S. 2010. Espaces politiques, espaces rituels : les bois sacrés de l'ouestCameroun Presses de Sciences Po, 3(55):19-38.

Mbayngone E, Thiombiano A, Hahn-hadjali K, Guinko S. 2008. Caractéristique écologique de la végétation ligneuse du sud-est du Burkina Faso (Afrique de l'ouest): le cas de la réserve de Pama. Candollea, 33(1): 17-33.

Mbayngone E. 2008. Flore et végétation de la réserve partielle de faune de Pama, sudest du Burkina Faso. Thèse de doctorat, Université de Ouagadougou, p.138.

Ouadba JM. 2003. Caractéristique de la végétation des milieux anthropisés de la province du Bazéga au Burkina Faso. Thèse d'Etat, Université de Ouagadougou, p.197.

Ouédraogo A. 2006. Diversité et dynamique de la végétation ligneuse de la partie orientale du Burkina faso. Thèse de doctorat de $3^{\text {ème }}$ cycle, Université de Ouagadougou, p.195.

Pielou EC. 1996. Species diversity and pattern diversity in the study of ecological succession. J. Theor. Biol., 370-383.

Sambaré O, Ouédraogo O, Wittig R, Thiombiano A. 2010. Diversité et écologie des groupements ligneux des formations ripicoles du Burkina Faso (Afrique de l'Ouest). Int. J. Biol. Chem. Sci., 4(5): 1782-1800.

Sanou BD. 2010. Aménagement des forêts de Dinderesso et du Kou et autoreprésentation des communautés villageoises riveraines. Proposition d'une méthode d'actualisation des coutumes pour une gestion durable des ressources naturelles. Bobo-Dioulasso, Burkina Faso.

SAS Institute Inc. 2008. JMP 8 Introductory Guide. Cary, NC, USA ISBN 978-159994-920-8. 
Savadogo S. 2008. Etude de la flore et de la végétation des bois sacrés de la zone subsahélienne du Burkina Faso. Mémoire de DEA, Université de Ouagadougou, p.65.

Savadogo S, Ouédraogo A, Thiombiano A. 2010. Perceptions, mode de gestion et végétation des bois sacrés au nord du Burkina Faso. Flora et Vegetatio SudanoSambesica, 13: 10-21.

Sinsin B. 1993. Phytosociologie, écologie, valeur pastorale, produit et capacité de charge des pâturages naturels du périmètre Nikki-Kalale au nord du Bénin. Thèse des sciences agronomiques, université libre de Bruxelles, p.389.

Sinsin B, Oumorou M. 2000. Etude de la diversité spécifique du groupement à Cochlospermum tinctorium A. Riche des savanes arbustives du nord Bénin. Acta. Bot. Gellica, 147(4): 345-360.

Sow M. 2003. Pratiques culturelles et conservation de la Biodiversité en Guinée. Education relative à l'environnement. Faculté des sciences, Université de Conakry, p.7.

Stevenson MG. 2005. Connaissance Traditionnelles et Gestion Durable des Forêts. Réseau de Gestion Durable des Forêts. Edmonton, Alberta.

Sukumaran S, Jeeva S. 2008. A floristic study on miniature sacred forests at Agastheeshwaram, southern peninsular India. Eur. Asian Journal of Biosciences, 2: 66-72.
Swamy PS, Kumar M, Sundarapandian SM. 2003. Spiritualité et écologie des bois sacrés au Tamil Nadu, Inde. Unasylva, 54(213): 53-58.

Thiombiano A. 2005. Les Combretaceae du Burkina Faso: Taxonomie, écologie, dynamique et régénération des espèces. Thèse de doctorat d'Etat, Université de Ouagadougou, p.290.

Traoré L. 2007. Bois sacrés, étude de la végétation ligneuse, mode de gestion dans les terroirs de Tiankuy, région de la boucle du Mouhoun. Mémoire de DEA, Université de Ouagadougou, p.50.

Traoré L, Ouédraogo I, Ouédraogo A, THIOMBIANO A. 2011. Perceptions, usages et vulnérabilité des ressources végétales ligneuses dans le Sud-ouest du Burkina Faso. Int. J. Biol. Chem. Sci., 5(1): 258-278.

Wilmanns O. 1989. Ökologische Pflanzensoziologie. 4. Aufl. -UTB 269. Quelles \& Meyer, Heindelberg.

Wite F. 1986. La Végétation de l'Afrique: Mémoire accompagnant la Carte de Végétation de l'Afrique. ORSTOM: Paris.

Yelkouni M. 2005. La gestion communautaire: une alternative pour la forêt de Tiogo au Burkina Faso. Revue d'Economie Régionale et Urbaine (RERU), 4: 557-578. 